\title{
CSF Pressure Change in Relation to Opening Pressure and CSF Volume Removed
}

\author{
(D) B. Griffith, (D)T. Capobres, (DS.C. Patel, (D) H. Marin, (D) A. Katramados, and (D).M. Poisson
}

\begin{abstract}
BACKGROUND AND PURPOSE: Idiopathic intracranial hypertension is a complex neurologic disorder resulting from increased intracranial pressure. Our aim was to determine whether a correlation exists between the CSF pressure-volume relationship, specifically the craniospinal elastance and pressure-volume index, in patients with idiopathic intracranial hypertension and whether opening pressure affects this relationship.
\end{abstract}

MATERIALS AND METHODS: Lumbar punctures performed for suspected idiopathic intracranial hypertension from 2006 to 2017 were identified. Opening and closing pressures, CSF volume removed, and clinical diagnosis of idiopathic intracranial hypertension were obtained from the medical records. The craniospinal elastance (pressure change per milliliter of CSF removed) and pressure-volume index were calculated, and the Pearson correlation coefficients between both the craniospinal elastance and pressure-volume index and opening pressure were determined. Linear regression models of craniospinal elastance and the pressure-volume index and interaction terms with opening pressure were assessed for covariate influence on this association.

RESULTS: One hundred sixteen patients were included in the final analysis. The mean craniospinal elastance according to opening pressure group was $0.52 \pm 0.18$ for $<20 \mathrm{~cm} \mathrm{H}_{2} \mathrm{O}, 0.57 \pm 0.20$ for $20-29 \mathrm{~cm} \mathrm{H}_{2} \mathrm{O}, 0.91 \pm 0.28$ for $30-39 \mathrm{~cm} \mathrm{H}_{2} \mathrm{O}$, and $1.20 \pm 0.25$ for $\geq 40$ $\mathrm{cm} \mathrm{H} \mathrm{H}_{2} \mathrm{O}$. There was a positive linear association between opening pressure and craniospinal elastance with a $0.28 \mathrm{~cm} \mathrm{H} \mathrm{H}_{2} \mathrm{O} / \mathrm{mL}$ increase in craniospinal elastance (standard error $=0.03, P<.001$ ) for every $10 \mathrm{~cm} \mathrm{H}_{2} \mathrm{O}$ increase in opening pressure. Of the covariables analyzed, only age older than 50 years and total volume of CSF removed affected this association.

CONCLUSIONS: As opening pressure increases, the craniospinal elastance increases in a linear fashion while the pressure-volume index decreases. Further studies are needed to determine whether these changes relate to the underlying pathophysiology of idiopathic intracranial hypertension or simply represent established CSF volume pressure dynamics.

ABBREVIATIONS: $\mathrm{BMI}=$ body mass index; $\Delta \mathrm{P} / \Delta \mathrm{V}=$ pressure change per milliliter of $\mathrm{CSF}$ removed; $E_{c S}=$ craniospinal elastance; $I \mathrm{CP}=$ intracranial pressure; $I I \mathrm{H}=$ idiopathic intracranial hypertension; $\mathrm{LP}=$ lumbar puncture; $\mathrm{OP}=$ opening pressure; $\mathrm{PVI}=$ pressure-volume index

diopathic intracranial hypertension (IIH) is a complex neurologic disorder resulting from increased intracranial pressure (ICP). Although the definite etiology of IIH is not known, disturbed CSF homeostasis, primarily due to impaired CSF absorption, and decreased craniospinal compliance have been implicated as possible causes of the elevated intracranial pressure. ${ }^{1,2}$

Although various criteria are needed to establish the diagnosis of IIH, documentation of an elevated ICP by lumbar puncture (LP) is required regardless of the criteria used. ${ }^{3,4}$ In addition to

Received November 2, 2017; accepted after revision February 24, 2018.

From the Departments of Radiology (B.G., T.C., S.C.P., H.M.), Neurology (A.K.), and Public Health Sciences (L.M.P.), Henry Ford Health System, Detroit, Michigan.

Please address correspondence to Brent Griffith, MD, Department of Radiology, K3, Henry Ford Hospital, 2799 West Grand Blvd, Detroit, MI 48202; e-mail: brentg@ rad.hfh.edu; @BrentDGriffith

http://dx.doi.org/10.3174/ajnr.A5642 helping establish the diagnosis of $\mathrm{IIH}$, the pressure and volume parameters acquired during the LP offer insight into the fluid dynamics of the craniospinal system. Specifically, pressure and volume changes can be used to determine craniospinal elastance $\left(E_{c s}\right)$, which represents the change in ICP per change in volume of CSF removed $(\Delta \mathrm{P} / \Delta \mathrm{V})$. In addition, the pressure-volume index (PVI) of the craniospinal system can be calculated. PVI represents the calculated volume required to raise the ICP by a factor of 10 and is directly proportional to the compliance of the CSF space. ${ }^{5}$

However, despite the frequency with which LPs are performed in patients with $\mathrm{IIH}$, no well-defined relationship has been established between the CSF volume removed and the change in ICP in patients with $\mathrm{IIH}$, and the few publications to date have shown varying results. One prior study evaluating patients younger than 22 years of age with suspected IIH found that ICP decreased by 1 $\mathrm{cm} \mathrm{H}_{2} \mathrm{O}$ for every $0.91 \mathrm{~mL}$ of CSF removed if the maximum 
change in pressure was $<15 \mathrm{~cm} \mathrm{H}_{2} \mathrm{O}{ }^{6}$ An additional study of 41 patients with IIH found that for every $1 \mathrm{~mL}$ of CSF removed, the closing pressure decreased by approximately $1.5 \mathrm{~cm} \mathrm{H}_{2} \mathrm{O}{ }^{7}$ However, neither of these studies accounted for changes in this relationship based on opening pressure (OP). More recently, Chisholm et $\mathrm{al}^{8}$ assessed $E_{c s}$ and PVI in patients with and without a diagnosis of IIH and found that there was a statistically significant association of both increased $E_{c s}$ and decreased PVI in patients with IIH. However, that study included only 20 patients with IIH. ${ }^{8}$

The primary purpose of this study was to evaluate pressure-volume relationships in a large series of patients with IIH undergoing LP, specifically $E_{c s}$ and PVI, as well as whether these relationships are affected by the initial opening pressure. These findings will be helpful in future studies assessing differences in patients with and without IIH.

\section{MATERIALS AND METHODS Lumbar Punctures}

This study was approved by the institutional review board. The imaging data base at our institution was searched for LPs performed between February 2006 and May 2017 with any of the following search terms: "idiopathic intracranial hypertension," "IIH," and "pseudotumor." This search yielded 304 LPs. The imaging records were reviewed to determine the OPs and closing pressures, volume of CSF removed, use of procedural sedation (usually performed by using a combination of up to $100 \mathrm{mcg}$ of fentanyl and $5 \mathrm{mg}$ of midazolam [Versed], which is titrated to patient comfort) and a decubitus-versus-prone positioning.

Pressure measurements and CSF volume removed were used to determine pressure change $\left(\Delta \mathrm{P}\right.$, centimeter $\left.\mathrm{H}_{2} \mathrm{O}\right)$ and pressure change per milliliter of CSF removed $(\Delta \mathrm{P} / \Delta \mathrm{V})$. LPs without all the required documentation and those performed with the patient in the prone position were excluded from analysis, resulting in 245 remaining lumbar punctures.

\section{Patients}

Patient charts for the remaining 245 LPs were reviewed to identify patient age, sex, body mass index (BMI), race/ethnicity, clinical diagnosis of IIH either before or following the LP, and the presence of CSF shunts at the time of the LP. Patients with CSF shunts and those without a clinical diagnosis of IIH were excluded. The clinical diagnosis of IIH was based on chart review and documentation of a diagnosis of IIH in the medical records. Specific clinical criteria used for establishing the diagnosis of IIH could not be determined due to the retrospective nature of the study. Additionally, in those patients with multiple LPs, only the first LP was included in the final analysis. The final study group included 116 patients.

\section{Statistical Analysis}

Calculation of craniospinal elastance $\left(E_{c s}\right)$ was performed according to established relationships $(\Delta \mathrm{P} / \Delta \mathrm{V})^{8,9}$ using $\mathrm{OP}$, closing pressure $(\mathrm{CP})$, and CSF volume removed $\left(\mathrm{mL}_{\mathrm{CSF}}\right)$ :

$$
E_{c s}=\mathrm{OP}-\mathrm{CP} / \mathrm{mL}_{\mathrm{CSF}}
$$

Calculation of the pressure-volume index (PVI), which represents the calculated volume required to raise the ICP by a factor of 10 , was performed according to the previously established equation using the same pressure and volume data:

$$
\mathrm{PVI}=\mathrm{mL}_{\mathrm{CSF}} / \log _{10}(\mathrm{OP} / \mathrm{CP})
$$

Continuous variables were summarized by median and range. Categoric variables were summarized by count and percentage. Analysis of variance, with post hoc 2-sample $t$ tests, was used to compare means between groups. A $\chi^{2}$ test was used to assess associations among categoric variables. Pearson correlation coefficients, with 95\% CIs, were estimated to express the correlation between CSF $\Delta \mathrm{P}$ and $\mathrm{OP}$ either in total or within subsets of patients.

Linear regression models of CSF $\Delta \mathrm{P}$ were developed using $\mathrm{OP}$, age (continuous or categoric), BMI (continuous or categoric), race (black or white), and sedation (yes or no) as potential predictors. Interaction terms with $\mathrm{OP}$ were used to determine whether a covariate (as continuous or categoric) had an influence on the association between OP and CSF $\Delta \mathrm{P}$. Sums of squares from nested models were used to construct $F$ tests to assess the contribution of additional variables to the model.

\section{RESULTS}

\section{Patient Demographics}

The study population included 116 patients; 109 (94.0\%) were female and $7(6.0 \%)$ were male with a median age of 33.5 years. In the 109 patients declaring race, $70(64.2 \%)$ were black and 39 (35.8\%) were white.

\section{Craniospinal Elastance in Relation to OP}

For purposes of assessing craniospinal elastance $\left(E_{c s}\right)$ as a function of $\mathrm{OP}$, patients were first categorized into 1 of 4 groups (OP of $<20,20-29,30-39$, and $\geq 40 \mathrm{~cm} \mathrm{H}_{2} \mathrm{O}$ ), depending on the OP (Fig $1 A)$. The mean $E_{c s}\left(E_{c s-m e a n}\right)$ according to the OP group was $0.52 \pm 0.18$ for an OP of $<20 \mathrm{~cm} \mathrm{H}_{2} \mathrm{O}, 0.57 \pm 0.20$ for an OP of $20-29 \mathrm{~cm} \mathrm{H}_{2} \mathrm{O}, 0.91 \pm 0.28$ for an OP of $30-39 \mathrm{~cm} \mathrm{H}_{2} \mathrm{O}$, and $1.20 \pm 0.25$ for an $\mathrm{OP}$ of $\geq 40 \mathrm{~cm} \mathrm{H}_{2} \mathrm{O}$ (Fig $1 A$ ). A 1-way analysis of variance of $E_{c s-m e a n}$ by group indicates that there was a statistically significant difference among the 4 groups ( $F$ test, $P<.001$ ). Additionally, there were significant differences in $E_{c s-m e a n}$ between any 2 groups ( $t$ test, $P<.005$ ), except for the 2 lowest groups ( $<20$ and $\left.20-29 \mathrm{~cm} \mathrm{H}_{2} \mathrm{O}\right)$.

When the OP was analyzed as a continuous measure rather than by group, there was a positive linear association between OP and $E_{c s}$ (Fig $1 B$ ). Specifically, when the $E_{c s}$ was regressed on OP, it was estimated that for every $10 \mathrm{~cm} \mathrm{H}_{2} \mathrm{O}$ increase in $\mathrm{OP}$, there was a $0.28 \mathrm{~cm}$ $\mathrm{H}_{2} \mathrm{O} / \mathrm{mL}$ increase in $E_{c s}$ (standard error $=0.03$, F test, $P<.001$ ). This finding demonstrated a moderately strong correlation, with the Pearson coefficient $=0.70(95 \% \mathrm{CI}, 0.59-0.78)$. With the predicted values from the linear regression of $E_{c s}$ regressed on OP, the expected $\Delta \mathrm{V} / \Delta \mathrm{P}$ relationship was estimated, demonstrating an exponential relationship with OP (Fig 1C). Specifically, at higher OPs, the volume of CSF required to be removed to achieve a similar change in pressure decreases with increasing OP.

\section{Pressure-Volume Index in Relation to OP}

For assessing the pressure-volume index as a function of OP, patients were again categorized into the same 4 OP groups (Fig 2A). The mean PVI per milliliter of CSF removed according to the OP 

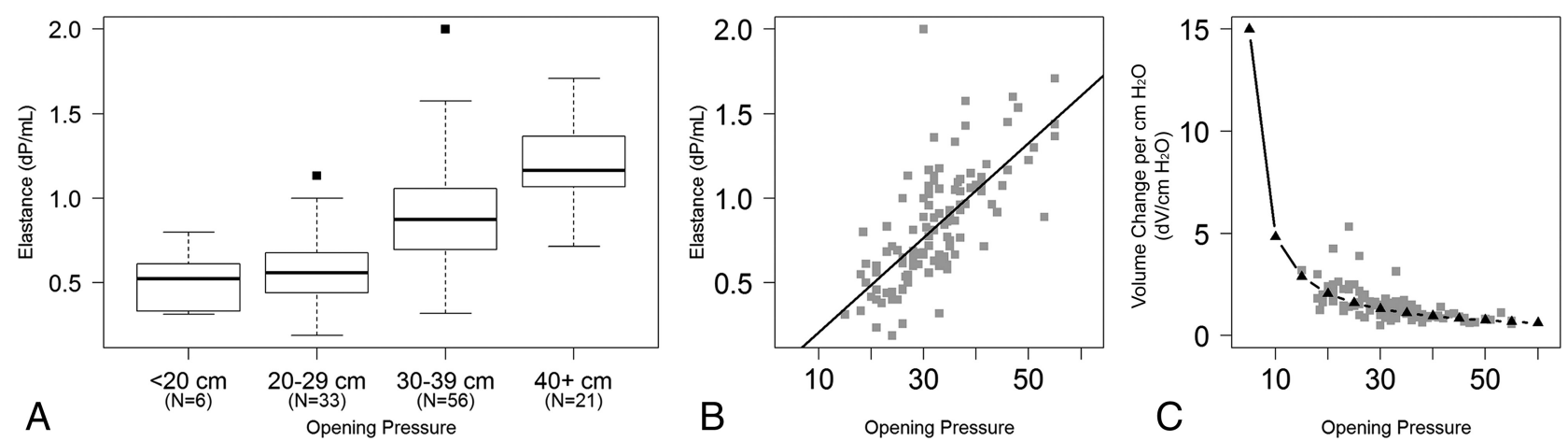

FIG 1. A, Boxplots depict the distribution of craniospinal elastance by increasing opening pressure. $B$, Scatterplot demonstrates $E_{c s}$ as a function of opening pressure using opening pressure as a continuous measure. The black line represents the linear regression model. $C$, Scatterplot demonstrates the inverse of elastance, which demonstrates the nonlinear relationship between the volume of CSF required to change the intracranial pressure as the opening pressure changes.
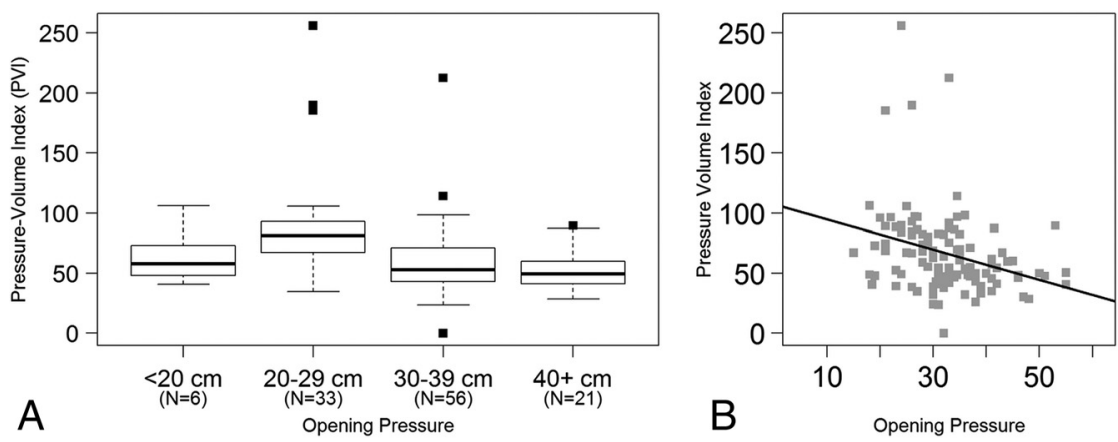

FIG 2. A, Boxplots depict the distribution of the pressure-volume index by increasing opening pressure. $B$, Scatterplot demonstrates PVI as a function of opening pressure using opening pressure as a continuous measure. The solid line represents the linear regression model.

group was $63.89 \pm 24.08$ for an $\mathrm{OP}$ of $<20 \mathrm{~cm} \mathrm{H}_{2} \mathrm{O}, 87.05 \pm 44.62$ for an OP of $20-29 \mathrm{~cm} \mathrm{H}_{2} \mathrm{O}, 59.79 \pm 30.21$ for an OP of $30-39 \mathrm{~cm}$ $\mathrm{H}_{2} \mathrm{O}$, and $51.97 \pm 15.74$ for an $\mathrm{OP}$ of $\geq 40 \mathrm{~cm} \mathrm{H}_{2} \mathrm{O}$ (Fig $2 \mathrm{~A}$ ). A 1 -way analysis of variance of PVI by group indicated that there was a statistically significant difference among the 4 groups ( $F$ test, $P<$.001). On further analysis, the significance was due to an increased mean in the $20-29 \mathrm{~cm} \mathrm{H}_{2} \mathrm{O}$ group compared with the 2 higher OP groups ( $t$ test, $P<.001$ ).

When the PVI was analyzed as a continuous measure rather than by group, there was a negative linear association between OP and PVI (Fig 2B). Specifically, PVI decreased by 1.25 for every 1 unit increase in OP (Fig 2B, solid line, standard error $=0.37, P=$ .001 , adjusted $\left.R^{2}=0.08\right)$. This finding demonstrated a significant negative correlation, with a Pearson coefficient of -0.30 (95\% CI, -0.46 to -0.13$)$.

\section{Covariable Analysis}

$E_{c s}$ and PVI in relation to OP were also assessed within each of the 4 covariate groups analyzed in this study: age, BMI, race/ethnicity, and use of procedural sedation (Table). Total CSF volume removed was also assessed as a covariable.

Age. The relationship between $E_{c s}$ and OP was not constant with age ( $F$ test of interaction $P=.03$ ). To examine this relationship, patients were grouped by age (younger than 30 years, $30-39$ years, $40-49$ years, and 50 years and older). It was estimated that for every $10 \mathrm{~cm} \mathrm{H}_{2} \mathrm{O}$ increase in $\mathrm{OP}$, there was a 0.27 to $0.34 \mathrm{~cm}$ $\mathrm{H}_{2} \mathrm{O} / \mathrm{mL}$ increase in $E_{c s}$ for the groups younger than 50 years of lationship (Table). There was evidence of a positive relationship between OP and PVI for patients 50 years and older with a 10.6 point increase in PVI per $10 \mathrm{~cm} \mathrm{H}_{2} \mathrm{O}$ increase in OP (coefficient $t$ statistic, $P=.02$ ). To assess whether these relationships were independent of OP, we evaluated for evidence of a correlation between age and $\mathrm{OP}$ or $\Delta \mathrm{P}$ and found no evidence of a correlation (Pearson coefficient with OP $=-0.14 ; 95 \% \mathrm{CI},-0.31-0.043$; Pearson coefficient with $\Delta \mathrm{P}=-0.06$; 95\% CI, $-0.24-0.12$ ).

BMI. BMI was recorded for 74 of the 116 cases in this study, and the median BMI was $42.0 \mathrm{~kg} / \mathrm{m}^{2}$. There was no evidence that BMI has any influence on the relationship between $E_{c s}$ and OP ( $F$ test of the interaction model, $P=.91 ; F$ test of the main-effects model, $P=.13$ ). There was no evidence that BMI has any influence on the relationship between PVI and OP ( $F$ test of the interaction model, $P=.84 ; F$ test of the main-effects model, $P=.39$ ). Cases without a recorded $\mathrm{BMI}$ had higher mean $\mathrm{OP}$ and $\Delta \mathrm{P}$ than those modeled here ( $t$ test, $P=.03$ and $P=.004$, respectively).

Race. There was no evidence that race has any influence on the relationship between $E_{c s}$ and OP ( $F$ test of the interaction model, $P=.85 ; F$ test of the main-effects model, $P=.82$ ). There was no evidence that race has any influence on the relationship between PVI and OP ( $F$ test of the interaction model, $P=.93 ; F$ test of the main-effects model, $P=.71$ ).

Sedation. There was no evidence that sedation has any influence on the relationship between $E_{c s}$ and OP ( $F$ test of the interaction 


\begin{tabular}{|c|c|c|c|c|c|}
\hline & \multirow[b]{2}{*}{ No. } & \multicolumn{2}{|r|}{$E_{c s}$} & \multicolumn{2}{|r|}{ PVI } \\
\hline & & Slope \pm SEM & Correlation Coefficient & Slope \pm SEM & Correlation Coefficient \\
\hline \multicolumn{6}{|l|}{ Age group (yr) } \\
\hline Younger than 30 & 39 & $0.34 \pm 0.04$ & $0.89(0.79-0.94)$ & $-20.1 \pm 5.9$ & $-0.48(-0.69$ to -0.20$)$ \\
\hline $30-39$ & 44 & $0.27 \pm 0.05$ & $0.67(0.47-0.81)$ & $-13.0 \pm 7.0$ & $-0.35(-0.58$ to -0.06$)$ \\
\hline $40-49$ & 24 & $0.28 \pm 0.06$ & $0.59(0.25-0.80)$ & $-10.5 \pm 8.5$ & $-0.24(-0.59$ to -0.18$)$ \\
\hline 50 and older ${ }^{b}$ & 9 & $0.10 \pm 0.08$ & $0.31(-0.44-0.81)$ & $10.6 \pm 11.7$ & $0.20(-0.54$ to 0.76$)$ \\
\hline \multicolumn{6}{|l|}{ BMI group $\left(\mathrm{kg} / \mathrm{m}^{2}\right)$} \\
\hline$<30$ & 8 & $0.44 \pm 0.14$ & $0.57(-0.23-0.91)$ & $-19.1 \pm 19.6$ & $-0.62(-0.92$ to 0.14$)$ \\
\hline $30-39$ & 20 & $0.20 \pm 0.07$ & $0.60(0.21-0.82)$ & $-2.7 \pm 9.3$ & $-0.11(-0.53$ to 0.35$)$ \\
\hline $40-49$ & 34 & $0.29 \pm 0.05$ & $0.79(0.62-0.89)$ & $-16.4 \pm 6.3$ & $-0.33(-0.60$ to 0.01$)$ \\
\hline$\geq 50$ & 12 & $0.20 \pm 0.08$ & $0.67(0.16-0.90)$ & $-1.6 \pm 10.5$ & $-0.08(-0.63$ to 0.51$)$ \\
\hline \multicolumn{6}{|l|}{ Race group } \\
\hline Black & 70 & $0.27 \pm 0.04$ & $0.69(0.54-0.80)$ & $-12.5 \pm 5.3$ & $-0.32(-0.52$ to -0.10$)$ \\
\hline White & 39 & $0.28 \pm 0.04$ & $0.66(0.44-0.81)$ & $-11.8 \pm 6.2$ & $-0.24(-0.52$ to 0.08$)$ \\
\hline \multicolumn{6}{|c|}{ Procedural sedation group } \\
\hline Yes & 46 & $0.28 \pm 0.05$ & $0.60(0.37-0.76)$ & $-19.0 \pm 6.5$ & $-0.32(-0.56$ to -0.04$)$ \\
\hline No & 70 & $0.28 \pm 0.03$ & $0.77(0.66-0.85)$ & $-10.2 \pm 4.5$ & $-0.34(-0.53$ to -0.11$)$ \\
\hline \multicolumn{6}{|c|}{ CSF volume removed (mL) } \\
\hline$<20$ & 27 & $0.38 \pm 0.06$ & $0.64(0.34-0.82)$ & $-14.0 \pm 10.3$ & $-0.27(-0.59$ to 0.12$)$ \\
\hline $20.0-24.9$ & 27 & $0.37 \pm 0.05$ & $0.79(0.59-0.90)$ & $-8.6 \pm 8.8$ & $-0.18(-0.53$ to 0.21$)$ \\
\hline $25.0-29.9$ & 30 & $0.38 \pm 0.05$ & $0.87(0.75-0.94)$ & $-18.3 \pm 8.7$ & $-0.53(-0.75$ to -0.21$)$ \\
\hline$\geq 30$ & 32 & $0.29 \pm 0.04$ & $0.90(0.80-0.95)$ & $-18.9 \pm 6.2$ & $-0.41(-0.67$ to -0.08$)$ \\
\hline
\end{tabular}

Note:-SEM indicates standard error of the mean.

${ }^{a}$ The slope indicates the average change in the measure for a $1 \mathrm{~cm} \mathrm{H} \mathrm{H}_{2} \mathrm{O}$ change in opening pressure. The Pearson correlation coefficient reflects the correlation between the measure and opening pressure.

${ }^{\mathrm{b}}$ Comparing 50 years and older with younger than 30 years $(P=.01), 30-39$ years $(P=.09)$, and $40-49$ years $(P=.08)$.

model, $P=.97 ; F$ test of the main-effects model, $P=.84)$. There was no evidence that sedation has any influence on the relationship between PVI and OP ( $F$ test of the interaction model, $P=.27$; $F$ test of the main-effects model, $P=.11$ ).

Volume Removed. There was some evidence that the volume of CSF removed influenced the slope of the linear relationship between opening pressure and $E_{c s}$ across all patients ( $F$ test of the interaction model, $P=.04)$. It was estimated that the increase in $E_{c s}$ with every $10 \mathrm{~cm} \mathrm{H}_{2} \mathrm{O}$ increase in OP is diminished by 0.068 points for every additional $10 \mathrm{~mL}$ removed (Table). To examine whether this relationship holds when linearity constraints are not in place, we grouped patients by volume removed $(<20 \mathrm{~mL}$, $20.0-24.9 \mathrm{~mL}, 25-29.9 \mathrm{~mL}$, and $\geq 30 \mathrm{~mL}$ quartiles). The interaction model did not show a significant change in slope across groups ( $F$ test of interaction model, $P=.42$ ). As a significant main effect ( $F$ test of the main effects model, $P<.0001$ ), volume removed modified the intercept of the line, which would be expected because both volume of CSF removed and $E_{c s}$ increase as the OP increases. There was evidence that the volume of CSF removed influenced only the intercept of the linear relationship between OP and PVI across all patients ( $F$ test of the interaction model, $P=.77 ; F$ test of the main effects, $P=.02)$. This finding was not retained within the quartiles of volume removed ( $F$ test of the interaction model, $P=.80 ; F$ test of the main effects, $P=.12$ ).

\section{DISCUSSION}

The results of this study demonstrate a clear relationship between change in ICP following LP and OP. Specifically, as the OP increases, the pressure change per milliliter of CSF removed (craniospinal elastance) increases in a linear fashion (Fig 1B). This linear relationship was present not only when comparing OP groups, but it also demonstrated a moderately strong correlation when considering $\mathrm{OP}$ as a continuous variable. When we assessed whether other potential factors, specifically age, BMI, race, use of procedural sedation, and total volume of CSF removed, may affect this relationship, only age older than 50 years and total volume of CSF removed were found to have any significant interaction. The interaction with age older than 50 years, though small, showed a decrease in average $E_{c s}$ as the OP increased. Similarly, there was a decrease in the strength of the relationship between $E_{c s}$ and $\mathrm{OP}$ as the volume of CSF removed increased, though this effect was removed when volumes were categorized into groups.

Because of the increasing craniospinal elastance at higher OPs, the volume of CSF required to be removed to achieve a similar change in pressure $(\Delta \mathrm{V} / \Delta \mathrm{P})$ decreases as the OP increases. In other words, the CSF volume $(\Delta \mathrm{V})$ required to be removed to cause a 1 unit change in CSF pressure is much smaller at higher OPs than at lower OPs. When this relationship between $\Delta \mathrm{V} / \Delta \mathrm{P}$ is plotted against $\mathrm{OP}$, a monoexponential relationship between $\Delta \mathrm{V}$ and $\Delta \mathrm{P}$ becomes evident (Fig $1 C$ ).

Similar to the $E_{c s}$, the pressure-volume index, which represents the calculated volume required to raise the ICP by a factor of 10 , also demonstrated a linear relationship with OP, though negative, which would be expected because PVI is a measure of system compliance and thus would be expected to decrease as OP increases. As with $E_{c s}$, there was again a difference noted for patients older than 50 years of age.

Three prior studies have investigated change in ICP according to CSF volume removed in patients with IIH. The largest of those, however, only evaluated patients younger than 22 years of age with suspected IIH. That study found that ICP decreased by $1 \mathrm{~cm}$ $\mathrm{H}_{2} \mathrm{O}$ for every $0.91 \mathrm{~mL}$ of CSF removed if the maximum change in pressure was $<15 \mathrm{~cm} \mathrm{H}_{2} \mathrm{O} .{ }^{6}$ However, if the $\Delta \mathrm{P}$ was beyond $15 \mathrm{~cm}$ $\mathrm{H}_{2} \mathrm{O}$, the relationship was no longer statistically significant. In 
addition, the study did not take into account the effect of differences in OP. The second study found that for every $1 \mathrm{~mL}$ of CSF removed, the closing pressure decreased by approximately $1.5 \mathrm{~cm}$ $\mathrm{H}_{2} \mathrm{O} .{ }^{7}$ However, that study assessed only 41 patients with preexisting diagnoses of IIH and, like the first study, did not account for the effect of different OPs. A more recent study by Chisholm et $\mathrm{al}^{8}$ assessed $E_{c s}$ and PVI in a group of 20 patients with IIH and 29 patients without IIH and found a statistically significant association of both increased $E_{c s}$ and decreased PVI in patients with IIH. That study did note the direct correlation between OP and $E_{c s}$ but found no effect of OP on PVI.

Elastance is a fundamental property of the craniospinal system. In the present study, craniospinal elastance was found to increase in a linear fashion as OP increased (Fig $1 B$ ). This linear correlation between $E_{c s}$ and $\mathrm{OP}$ is then responsible for the monoexponential shape of the volume-pressure curve (Fig 1A). These findings are expected. In fact, in 1975, Marmarou et $\mathrm{al}^{5}$ demonstrated a similar relationship in experiments performed on cats. That study found that at low initial pressure levels, large increases in fluid volume resulted in relatively small increases in ICP. ${ }^{5}$ In contrast, at high initial pressures, the compliance of the system is reduced and small volume additions resulted in larger increases in pressure. ${ }^{5}$ When plotted on a linear axis, the volume-pressure curve in that study was found to be exponential, with the slope $(\Delta \mathrm{V} / \Delta \mathrm{P})$ decreasing as the pressure increased. ${ }^{5}$ Although our study looked at the inverse of this volume-pressure curve relationship, with fluid being removed from the system rather than added, our findings support this monoexponential volume-pressure model.

In 1979, Avezaat et $\mathrm{al}^{9}$ performed a similar study in anesthetized and ventilated dogs at changing ventricular fluid pressures. That study assessed the volume-pressure response, which is defined as the immediate change in ICP resulting from a uniform change in ventricular CSF volume and is also a measure of intracranial elastance. ${ }^{9}$ Most interesting, in contrast to the mono-exponential volume-pressure model described by Marmarou et al, ${ }^{5}$ Avezaat et $\mathrm{al}^{9}$ actually described 3 distinct zones in this relationship. In zone 1, the volume-pressure response, or elastance, increased linearly with the ventricular fluid pressures, which would be in accordance with the monoexponential model described by Marmarou et al. ${ }^{5}$ However, in zone 2, the volume-pressure response leveled off or even decreased, and finally, at the advanced stage of cerebral compression, zone 3 , the volume-pressure response started to rise again, but more rapidly than during zone $1 .{ }^{9}$ The study concluded that the exponential volume-pressure model is valid for a restricted ICP range only and theorized that the breakpoint in the monoexponential relationship may relate to failure of autoregulation, given the role that intracranial hemodynamics play in determining ICP. ${ }^{9}$ Although the findings of our study fit the monoexponential model put forth by Marmarou et $a l,{ }^{5}$ it would be difficult to say for certain that the different zones identified by Avezaat et $\mathrm{al}^{9}$ do not exist because these changes may take place outside the physiologic range of CSF pressures seen in patients with IIH.

The other interesting finding in this study is the effect of older age on $E_{c s}$ and PVI, a finding only seen in patients older than 50 years of age. Although the cause for this interaction cannot be ascertained from this study, it is possible that age-related brain volume loss counteracts the effect of increasing OP, thereby dampening the effect on $\Delta \mathrm{P}$. However, with only 9 patients older than 50 years of age, these relationships require further study for validation.

Of course, because this study assessed only patients with IIH, it is not possible to assess whether these findings are due to pathophysiologic changes associated with IIH or simply reflect the expected changes in the normal craniospinal system as the pressure of the system increases. To that end, 1 recently published study similar to this one compared measures of elastance and PVI in patients with and without $\mathrm{IIH}^{8}$ That study found that patients with IIH had higher elastance and lower PVI and suggested that this increased elastance may contribute to the pathophysiology of IIH. However, that study was much smaller, including only 20 patients with $\mathrm{IIH}$, making comparison between the 2 groups more difficult, given the influence that OP has on these parameters. That study also found that PVI was independent of OP. However, in this study, although the strength of the correlation was less, there was a negative linear association between PVI and OP.

The greatest limitation of our study is that only opening and closing pressures were obtained. As a result, while the value obtained for $E_{c s}$ is ascribed to a single OP, in fact, this value is likely continuously changing as fluid is being removed and the ICP is decreasing. Thus, the $E_{c s}$ calculated for higher OPs is likely blunted by an averaging effect from the $E_{c s}$ as fluid is being removed at lower pressures. This feature likely accounts for the effect that the volume of CSF removed had on the relationship between $E_{c s}$ and OP, with the increase in $E_{c s}$ for every $10 \mathrm{~cm} \mathrm{H}_{2} \mathrm{O}$ increase in OP diminished by 0.068 points for every additional 10 $\mathrm{mL}$ removed. It is thus possible that the true $E_{c s}$ may actually be higher than this study suggests at higher OPs. Thus, a confirmatory study measuring pressure change at multiple time points during the removal of CSF would be of value.

The lack of data points at lower OPs is also a limitation, thus requiring an estimation for $\Delta \mathrm{V} / \Delta \mathrm{P}$ at low OPs based on the slope of the $E_{c s}$. The study was also limited by its retrospective nature. Because a clinical diagnosis of IIH was determined by chart review, it is possible that some patients were inappropriately included or excluded. An additional limitation was the inclusion of patients undergoing treatment for IIH. While patients with CSF shunts were excluded and only the first LPs were included when multiple LPs were available, some patients were likely receiving pharmacologic treatment for IIH at the time of the LP. This might explain the small number of patients with a clinical diagnosis of $\mathrm{IIH}$, but normal CSF OPs. In addition, while the measurement and documentation of CSF pressures and volume of CSF removed are objective by nature, techniques vary by proceduralist, which could add a degree of variability to the results.

\section{CONCLUSIONS}

This study demonstrates the effect of OP on craniospinal system fluid dynamics, specifically $E_{c s}$ and PVI, in patients with IIH undergoing LP. Recent studies suggest that these dynamics differ in patients with IIH, which may provide insight into the disease pathogenesis. However, further studies comparing the results of this study with similar measurements in patients without IIH are 
needed to determine whether these changes reflect expected variations due to normal changes in craniospinal elastance or may be related to pathophysiologic changes in IIH.

\section{REFERENCES}

1. Tain RW, Bagci AM, Lam BL, et al. Determination of cranio-spinal canal compliance distribution by MRI: methodology and early application in idiopathic intracranial hypertension. J Magn Reson Imaging 2011;34:1397-404 CrossRef Medline

2. Alperin N, Ranganathan S, Bagci AM, et al. MRI evidence of impaired CSF homeostasis in obesity-associated idiopathic intracranial hypertension. AJNR Am J Neuroradiol 2013;34:29-34 CrossRef Medline

3. Friedman DI, Liu GT, Digre KB. Revised diagnostic criteria for the pseudotumor cerebri syndrome in adults and children. Neurology 2013;81:1159-65 CrossRef Medline

4. Headache Classification Committee of the International Headache Soci- ety. The International Classification of Headache Disorders, 3rd edition (beta version). Cephalalgia 2013;33:629-808 CrossRef Medline

5. Marmarou A, Shulman K, LaMorgese J. Compartmental analysis of compliance and outflow resistance of the cerebrospinal fluid system. J Neurosurg 1975;43:523-34 CrossRef Medline

6. McLaren SH, Monuteaux MC, Delaney AC, et al. How much cerebrospinal fluid should we remove prior to measuring a closing pressure? J Child Neurol 2017;32:356-59 CrossRef Medline

7. Fiorito-Torres F, Rayhill M, Perloff M. Idiopathic intracerebral hypertension (IIH)/pseudotumor: removing less CSF is best. Neurology 2014;82(10 Suppl):I9-1.006

8. Chisholm JT, Sudhakar P, Alhajeri AN, et al. Intracranial elastance is increased in idiopathic intracranial hypertension. Eur J Neurol 2017; 24:1457-63 CrossRef Medline

9. Avezaat CJ, van Eijndhoven JH, Wyper DJ. Cerebrospinal fluid pulse pressure and intracranial volume-pressure relationships. J Neurol Neurosurg Psychiatry 1979;42:687-700 CrossRef Medline 